\title{
Comparative analysis of dust emissions: isolated stockpile vs two nearby stockpiles
}

\author{
B. Furieri ${ }^{1}$, S. Russeil ${ }^{1}$, J.-L. Harion ${ }^{1}$, J. Santos ${ }^{2} \&$ M. Milliez ${ }^{3}$ \\ ${ }^{1}$ Department of Industrial Energetics, Ecole des Mines de Douai, France \\ ${ }^{2}$ Department of Environmental Engineering, \\ Federal University of Espirito Santo, Brazil \\ ${ }^{3} E D F R \& D$, France
}

\begin{abstract}
The estimation of dust emission of fugitive sources, suchas storage piles of granular materials on industrial sites, is often carried out by implementing mathematical models. The widely used USEPA model employs emission factors which require a detailed knowledge of the fluid flow. The present work is focused on the investigation of global emissions from oblong stockpiles. Previous studies dealt with dust emissions quantification and fluid flow analysis concerning isolated oblong piles. Later work indicated the importance of emissions originating from the ground region surrounding piles whose contribution can be up to twice that coming from the pile. In this framework the stockpile oriented $60^{\circ}$ to the main wind flow direction has presented the most elevated global dust emission rate. This paper aims to analyse the influence of a second successive pile on global dust emissions on industrial sites, on which there is rarely a single isolated pile. Dust emission rates are assessed by the USEPA model and fluid flow data is predicted by numerical simulations carried out with the open-source CFD code Code_Saturne. The distance between piles is also a parameter analysed in these investigations. The following configurations have been carried out: (i) one isolated stockpile and (ii) two parallel stockpiles with the same orientation and two different values of gap between them. The investigation of the two successive nearby stockpiles indicates that dust emission from the upstream stockpile is slightly increased when the piles are more distant. On the contrary, the analysis of the downstream stockpile shows that, as the distance increases, dust emission will be decreased. It is also found that the isolated stockpile emits less dust than the upstream or
\end{abstract}


downstream pile. Global emissions are found to be approximately the same for the two distances between piles that have been tested.

Keywords: dust emission quantification, USEPA model, CFD, Code_Saturne.

\section{Introduction}

The investigation of aeolian erosion of diffuse sources is a subject of major importance as we are interested in the reduction of environment degradation. Even so, some authors dedicated their works to the quantification and discussions about dust emissions and the development of quantification methodologies [2-7]. These authors focused their works on open storage yards of granular material. Badr and Harion [2] investigated the influence of wind flow conditions and pile dimensions (height and width) on dust emission rates. Toraño et al. [4] also carried out numerical simulations to study the influence of pile shapes and fluid flow on wind erosion. These authors found a strong influence of the wind flow on the typical fluid flow structures around a pile and consequently on the dust emission rates. Toraño et al. [4] also stated that a semicircular stockpile shape corresponds to lower emission rate when compared to conic and flat-topped stockpile shapes. Diego et al. [5] carried out an implementation of a mathematical model of dust emission quantification in a commercial CFD package. They investigated a configuration of parallel stockpiles and found out that the upstream pile works as a protection to the other pile. Later work of Toraño et al. [6] showed the influence of wind barriers on dust emissions from storage piles by using numerical simulation and the USEPA model. Their study has shown a reduction of about $66 \%$ on dust emission caused by the barriers. Turpin and Harion [3] based their work on the impact of the stockpile crest on the overall dust emission. Several clipping heights of flat-topped piles were examined to determine their impact on dust emission. The main conclusion was that the flattening of stockpile's crest does not reduce the pollution. Thus, oblong stockpiles with sharped crest were found as emitting the smallest amount of dust. After that, Turpin and Harion [7] investigated the influence of nearby buildings on dust emissions from stockpiles of real industrial sites. The complex configuration of these sites was simulated: three stockpiles and several rectangular and cylindrical buildings. The remarkably influence of the obstacle on the total stockpile dust emissions was highlighted by these results.

All these works were carried out employing the USEPA model [1]. This methodology is devoted to the quantification of dust emissions from diffuse sources. In a few words, the USEPA model formulation is highly dependent on the fluid flow pattern on the surface. Furthermore, the model is based on the near wall velocity distribution and numerical simulations with good resolution is required to analyse the amount of dust emitted from several classes of piles, wind flow condition and materials.

As previously observed, the investigation of oblong stockpiles was only carried out with one isolated stockpile [3]. However, on industrial sites there is rarely a single isolated stockpile. The stockpiles are organized one nearby the others, usually parallel. As the quantification is deeply dependent on the velocity 
distribution over the surface of interest this paper deals with the modifications observed if a second pile is considered downstream the first one. Moreover, the influence of the gap between piles on the fluid flow is analysed and consequently the emissions. The main objective of this paper is to compare the dust emitted from an isolated stockpile and from two nearby stockpiles. For this paper, only a stockpile oriented $60^{\circ}$ to main wind flow direction is analysed.

\section{Numerical simulation background}

The solution of the three dimensional equations of mass and momentum was carried out with the open-source Code_Saturne [8]. Figure 1(a) shows the computational domain and the boundary conditions and Figure 1(b) indicates the meshing used in the present numerical simulations. The dimensions of the real stockpile were scaled-down by a factor of 1:200 to carry out the numerical simulations: $0.578 \mathrm{~m}$ (width), $0.205 \mathrm{~m}$ (length) and $0.08 \mathrm{~m}$ (height). The model is an oblong stockpile with an angle of repose equal to $38^{\circ}$ which is representative of coal piles commonly found in real industrial sites. Dimensions of upstream and downstream zones were chosen to ensure that the results are not influenced by the size of the domain (as performed by Turpin and Harion [3] for a same pile). Spanwise and vertical dimensions correspond to the wind-tunnel dimensions on which this study is based on. In fact, the vertical dimension of the computation domain was set as half of the wind-tunnel height.

Turbulence effects were accounted for by using the k- $\omega$ Shear Stress Transport (SST) model without wall functions for the turbulence damping near walls. As required for the use of this turbulent model without wall functions there is a mesh refinement near the wall surface where $z^{+} \leq 5$. The mesh is produced by an extrusion from triangular cells defined on the ground surface and pile walls (see Figure 1(b)) towards the top boundary of the computational domain. Mesh refinement and density were set based on the last numerical studies with oblong

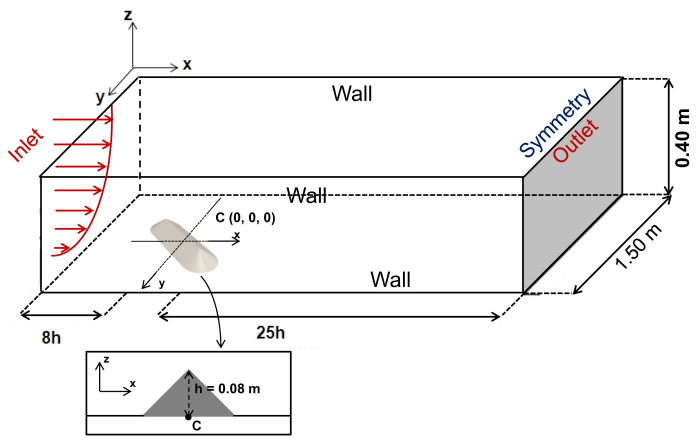

(a)

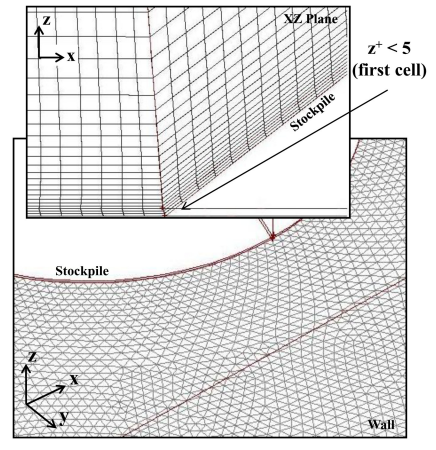

(b)

Figure 1: Schematic configuration of the computational domain. 
stockpiles successfully validated by PIV experiments (see Badr and Harion [9] and Turpin [10]).

The inlet boundary conditions for velocity $(u, v$ and $w)$, turbulent kinetic energy $(k)$ and specific dissipation rate $(\omega)$ were obtained from previous numerical simulations of a flow in a channel with the same dimensions (height and width) of the computational domain used in the present work. In these previous simulations, a periodic streamwise flow was set to produce a fully developed channel flow and the converged flow field is set as the inlet condition for the present simulation. The free stream velocity $\left(u_{r}\right)$ tested was equal to $6.5 \mathrm{~m} / \mathrm{s}$. For the outlet boundary conditions, it is assumed that the flow is fully developed and for the upper boundary condition, symmetry was imposed. Finally, smooth walls with no-slip conditions are set at domain lateral walls, as well, at stockpile and ground walls.

\section{USEPA model to dust emission quantification}

Figure 2 presents the USEPA model [1] under an algorithm form. The model requires the following input data to dust emission quantification: particle size multiplier (taken as the average particles size) $(k)$, threshold friction velocity $\left(u_{t}^{*}\right)$, distribution of $u_{s} / u_{r}$ on the surface of interest $\left(u_{r}\right.$ is the reference velocity at $10 \mathrm{~m}$ height from the ground and $u_{s}$ is the velocity at $25 \mathrm{~cm}$ from the surface, both for real scale), number of perturbations ( $\mathrm{N})$, number of isosurfaces of $u_{s} / u_{r}$ (M) and the highest velocity value measured by an anemometer at a reference height for a period between perturbations $\left(u_{10}^{+}\right)$. The whole input data set is issue from the materials and industrial site characteristics, excepting the distribution of the ratio $u_{s} / u_{r}$ which needs a numerical simulation of the impinging wind flow. CFD calculations were carried out with the open-source "Code_Saturne" to obtain $u_{s} / u_{r}$ distribution on the surfaces of interest. One perturbation per year was considered for the configurations investigated. A perturbation is defined in the USEPA guidance [1] as an intervention done in a storage pile yard for maintenance and transport of material.

Equation 1 is the fundamental formulation of USEPA methodology to calculate the emission rate $(\mathrm{E})$. The pile surface is divided in subareas representing the different levels of wind erosion exposure (values of the ratio $u_{s} / u_{r}$ ). Each subarea is considered as a distinct source corresponding to a constant value of $u_{s} / u_{r}$. Thus, the summation in Equation 1 considers these subareas separately with the respective fraction of subarea $S$.

$$
E=k \sum_{i=1}^{N} \sum_{j=1}^{M} P_{i j} S_{i j}
$$

where $P$ is the erosion potential $\left(\mathrm{g} / \mathrm{m}^{2}\right)$.

The erosion potential $P$ is calculated based on the friction velocity $\left(u^{*}\right)$ and threshold friction velocity $\left(u_{t}^{*}\right)$ as shown in Equation 2 . For the disturbed fluid flow around stockpiles the USEPA model proposes Equation 3 to determine 


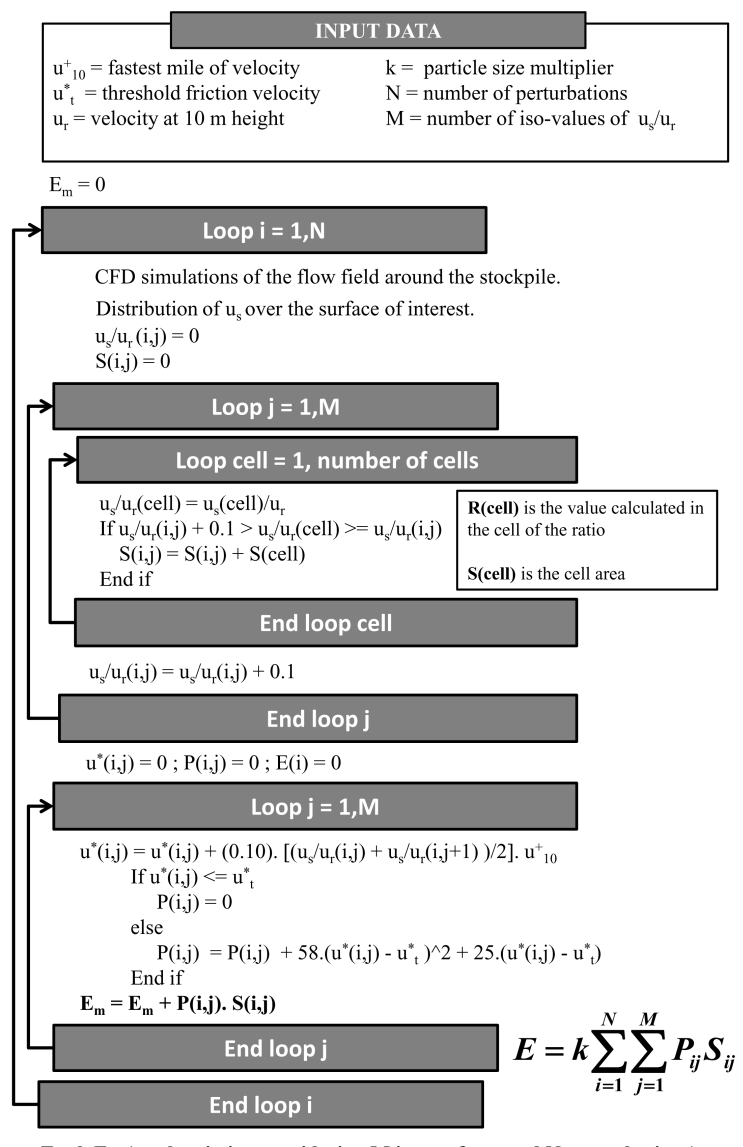

$E=k . E_{m}$ (total emission considering $M$ iso-surfaces and $N$ perturbations)

Figure 2: USEPA model algorithm.

friction velocity.

$$
\begin{gathered}
\left\{\begin{array}{c}
P=58\left(u^{*}-u_{t}^{*}\right)^{2}+25\left(u^{*}-u_{t}^{*}\right) \\
P=0 \text { for } u^{*} \leq u_{t}^{*}
\end{array}\right. \\
u^{*}=0.10\left(\frac{u_{s}}{u_{r}}\right) u_{10}^{+}
\end{gathered}
$$

The aerodynamic particle size multiplier $(k)$ is different for each aerodynamic particle size considered (cf. Section 13.2.5.3 in USEPA guide [1]). The value of $k$ equal to 0.5 was chosen to represent $P M_{10}$ emissions. The value of $0.35 \mathrm{~m} / \mathrm{s}$ for coal on the stockpile surface was determined from wind-tunnel experiments (cf. Turpin and Harion [3]). 
290

Air Pollution XX

4 Wall flow topology on stockpile surface

This work aims to demonstrate that the arrangement of a second successive stockpile substantially modifies the wall flow topology on their surfaces. Even the upstream stockpile, where the wind flow impinges, indicates a considerable different (compared to the isolated pile configuration) wall flow topology. Thus, Figures 3(a), 3(b) and 3(c) illustrate the configurations studied and contours of the ratio $u_{s} / u_{r}$ plotted on the surface distant $25 \mathrm{~cm}$ (real scale) from the stockpile wall. The gap between piles is a function of the parameter $e$ which is equal to $0.894 h$ ( $h$ is the stockpile height).

The near wall flow distribution of the isolated stockpile (Figure 3(a)) and two nearby stockpiles (Figures 3(b) and 3(c)) presents some distinctions. The results of two nearby stockpiles configurations indicate that the presence of a downstream stockpile highly modifies the fluid flow pattern of the upstream one, especially

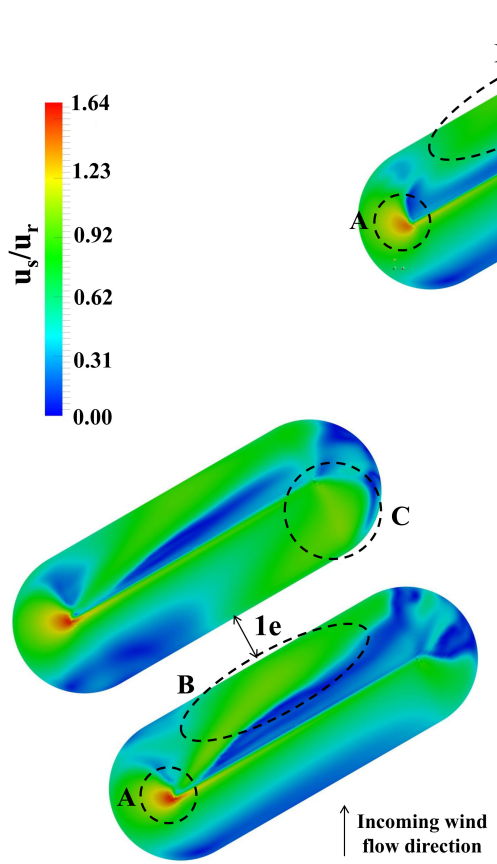

(b)

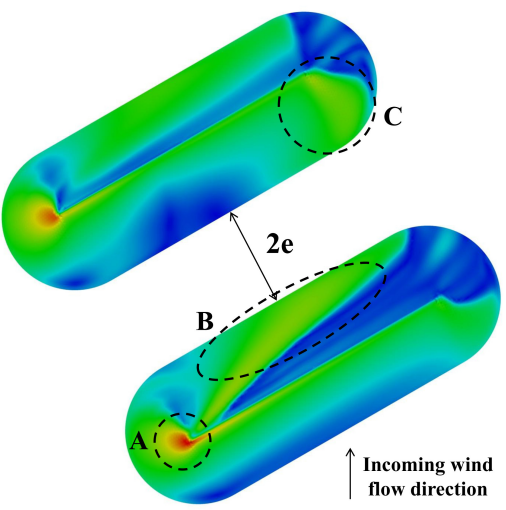

(c)

Figure 3: Wall flow topology of stockpiles oriented $60^{\circ}$ to the main wind flow direction: (a) isolated stockpile, (b) two stockpiles with a gap equal to $1 e$ and (c) two stockpiles with a gap equal to $2 e$.

WIT Transactions on Ecology and The Environment, Vol 157, (C) 2012 WIT Press www.witpress.com, ISSN 1743-3541 (on-line) 


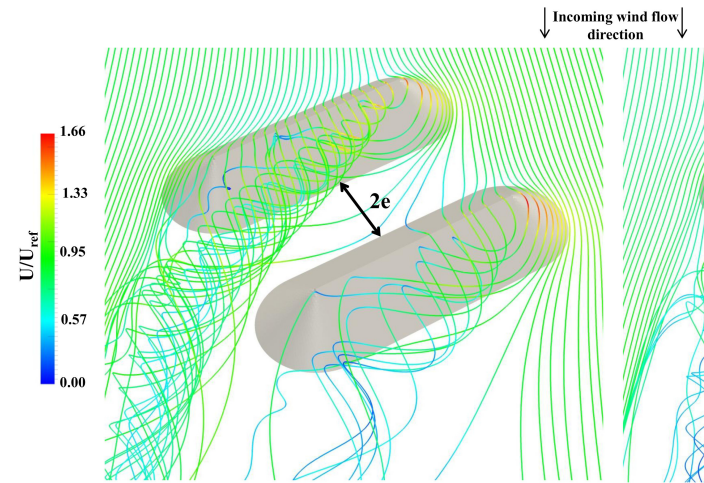

(a)

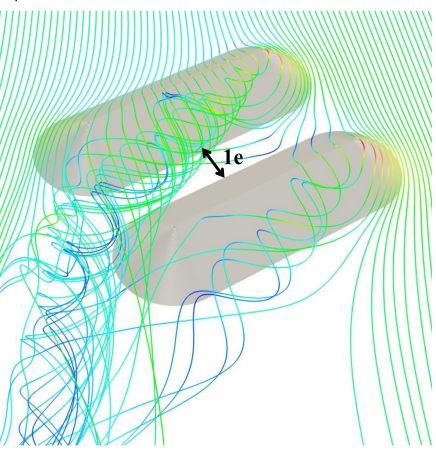

(b)

Figure 4: Streamlines coloured by the wind flow velocity magnitude: (a) two stockpiles with a gap equal to $2 e$ and (b) two stockpiles with a gap equal to $1 e$. (The velocity measured at $10 \mathrm{~m}$ in real scale is chosen as the reference velocity $U_{\text {ref }}=5.26 \mathrm{~m} / \mathrm{s}$.)

on the leeward side. Furthermore, the following common characteristics may be listed: (i) on the impingement point of the upstream stockpile there is a zone of high levels of $u_{s} / u_{r}$ up to 1.6 , and on the isolated stockpile the maximum value reaches 1.3 (zones A in Figure 3), (ii) on the leeward side of upstream stockpile there are wide zones of $u_{s} / u_{r}$ in the range 1.2-1.3 (zones B in Figure 3), otherwise on the isolated pile this value is smaller than 0.9 and (iii) the right side of the downstream pile (see highlighted zones $\mathrm{C}$ in Figure 3 ) indicates more zones of high values of $u_{s} / u_{r}(\approx 1.2)$. In addition, on the downstream stockpile of the configuration with gap equal to $1 e$ the least amount of low values of the ratio $u_{s} / u_{r}$ are noticed. It is worth to note that, smaller is the gap between piles, greater is the effect of the disturbed fluid flow on the stockpile surface which may increase wind erosion.

Figures 4(a) and 4(b) present the pathlines over the nearby stockpiles, respectively, with gap equal to $2 e$ and $1 e$. Supported by these images we can extend the explanations given in the previous paragraph about the modification of the mean fluid flow pattern due to the presence of a second nearby stockpile. Indeed, comparing Figures 4(a) and 4(b) the influence of the gap is clearly observed. Figure 4(a), representing the gap $2 e$, shows that the main vortex developing downstream the first pile does not impinge the downstream pile. Only few modified streamlines have influence on the pile as would have the free stream flow impinging an isolated pile. On the contrary, Figure 4(b) (gap 1e) shows that streamlines impinge on the windward wall of the downstream pile increasing the amount of surface having important values of $u_{s} / u_{r}$ as may also be observed in Figure 3. 


\section{Dust emission quantification: isolated vs two nearby stockpiles}

This section compiles the results of dust emission calculated with the USEPA model. Table 1 and Table 2 show these results of dust emission quantification for the isolated stockpile and two nearby stockpiles with a gap equal to $2 e$ and $1 e$. Three values of wind flow velocity $(5,10$ and $15 \mathrm{~m} / \mathrm{s})$ were also considered in the analysis of dust emission quantification. Velocity values between 5 and $10 \mathrm{~m} / \mathrm{s}$ were chosen as the most representative of usual wind velocities. The influence of higher velocity values is also analysed by the calculation of dust emission for $15 \mathrm{~m} / \mathrm{s}$. Table 1 presents the dust emitted from each pile separately and Table 2 the summation and respective relative difference of twice the dust quantified of an isolated pile compared to the sum of each stockpile in the configuration of two nearby stockpiles.

Results of dust emission quantification are a direct consequence of the wall flow topology. Analysis of Table 1 leads to the following conclusions about dust quantification: (i) the amount of particles quantified for the isolated stockpile is smaller than the upstream or downstream pile in the configuration with two nearby piles whatever the velocity is, (ii) the difference of dust emission between upstream and downstream pile is smaller as the gap increases and (iii) the downstream pile emits more particles for all velocities tested for the configuration with gap 1e. For the configuration with gap equal to $2 e$, the downstream emission is greater than upstream for the highest velocity value $\left(u_{10}^{+}\right)$higher than approximately $13 \mathrm{~m} / \mathrm{s}$ (several values of $u_{10}^{+}$were tested to obtain this conclusion however only three are presented herein). For smaller velocities, the upstream emission is always slightly smaller.

Table 1: Dust emissions quantification of each pile.

\begin{tabular}{|c|c|cc|cc|}
\cline { 2 - 6 } \multicolumn{1}{c|}{} & \multicolumn{5}{c|}{ Dust emission [kg/year] } \\
\hline & & Two $(2 e)$ & Two (2e) & Two (1e) & Two (1e) \\
$u_{10}^{+}$ & Isolated & upstream & downstream & upstream & downstream \\
\hline 5 & 2.1 & 3.1 & 2.6 & 2.6 & 3.5 \\
10 & 31.3 & 34.8 & 34.5 & 33.0 & 42.4 \\
15 & 91.2 & 97.9 & 98.7 & 94.7 & 118.5 \\
\hline
\end{tabular}

Table 2: Sum and relative difference between the tested cases.

\begin{tabular}{|c|c|cc|cc|}
\cline { 2 - 6 } \multicolumn{1}{c|}{} & & \multicolumn{2}{c|}{ Two (2e) } & \multicolumn{2}{c|}{ Two (1e) } \\
\hline & Twice isolated & \multicolumn{2}{c|}{ upstream and downstream } & upstream and downstream \\
\hline$u_{10}^{+}$ & $\begin{array}{c}\text { Dust emission } \\
{[\mathrm{kg} / \text { year }]}\end{array}$ & $\begin{array}{c}\text { Dust emission } \\
{[\mathrm{kg} / \mathrm{year}]}\end{array}$ & $\begin{array}{c}\text { Difference } \\
{[\%]}\end{array}$ & $\begin{array}{c}\text { Dust emission } \\
{[\mathrm{kg} / \mathrm{year}]}\end{array}$ & $\begin{array}{c}\text { Difference } \\
{[\%]}\end{array}$ \\
\hline 5 & 4.2 & 5.7 & 35.7 & 6.1 & 45.2 \\
10 & 62.6 & 69.3 & 10.7 & 75.4 & 20.4 \\
15 & 182.4 & 196.6 & 7.8 & 213.2 & 16.9 \\
\hline
\end{tabular}


The purpose of Table 2 is to find out practical implication for the piles distribution on real industrial sites. For both gaps considered, the sum of the emission quantified for each stockpile in the configuration with two nearby stockpiles is greater than twice the isolated pile case. This difference in the quantification of dust emission has a maximum value of $45.2 \%$ for the smallest tested velocity. This is a significant result, as this wind velocity value is the most usual noticed in nature.

The differences in dust quantification for the tested configurations are more important as the main velocity value decreases. This behaviour may be explained by the distribution of erodible and non-erodible surface area (parameter which defines if the erosion potential is null, cf. Equation 2). The greatest deviations of surface distribution, between isolated pile and upstream and downstream pile, is observed for $u_{s} / u_{r}$ values greater than 0.85 . Finally, a small value of $u_{10}^{+}$, which results in small values of $u^{*}$ (Equation 3) only indicates dust emission for $u_{s} / u_{r}$ values greater than 0.75 (cf. Equation 1). Thus, the differences in the distribution of $u_{s} / u_{r}$ are more perceived for small values of $u_{10}^{+}$. If the quantification of dust emission is carried out using a high value of $u_{10}^{+}$the deviation is less perceived in the final amount of particles quantified. Such affirmation is confirmed by second and sixth columns of Table 1 which indicate a difference of dust quantification up to approximately $60 \%$ for the velocity equal to $5 \mathrm{~m} / \mathrm{s}$.

\section{Conclusions}

This work focuses on the analysis of the effect of a second successive pile on global dust emissions. The USEPA model was chosen as the methodology to quantify dust emissions. The USEPA model application depends on some input data concerning the pile and its environment. The most significant input data is the wind velocity distribution near the surface of interest. This distribution was obtained with a good resolution by numerical simulations using the open-source CFD software Code_Saturne.

CFD calculations have shown significant differences of the near wall velocity distribution between isolated and two successive piles oriented $60^{\circ}$ to the incoming air flow. There was also a differentiation caused by the gap between the two nearby stockpiles. Our results have shown that on industrial sites the total dust emission from two nearby stockpiles is underestimated if the quantification is taken as twice the emission calculated for an isolated stockpile. Results have shown an augmentation of dust emission quantification up to $45.2 \%$ for a given velocity if it is considered the sum of each pile in a configuration with two nearby stockpiles. The total of dust emission from two nearby stockpiles increases as the gap between piles decreases. This may be interpreted by a stronger influence of the main vortex (produced in the upstream stockpile) on the downstream stockpile as they are at a shorter distance. The simulation of others incoming wind flow directions are scheduled to complete these analysis.

The main practical application of the USEPA model is the quantification in real scale of the amount of dust emitted on industrial sites. Thus, as these 
quantifications are highly dependent on numerical calculations the kind of investigation presented in this work are often carried out. To that aim, a software called Atmospheric Diffuse Dust Emissions on Industrial Sites (ADDEmIS) [11] was developed for the functional quantification of dust emissions on industrial sites. It considers a database containing the several existing class of diffuse sources on a given industrial site and corresponding fluid flow pattern issue of previous numerical simulations. Using this software would enable to analyse other configurations and wind flow directions.

\section{Acknowledgement}

This work was carried out with the financial support of EDF R\&D.

\section{References}

[1] USEPA, Miscellaneous Sources, Industrial Wind Erosion, AP-42, 2006.

[2] Badr, T. \& Harion, J.L., Effect of aggregate storage piles configuration on dust emissions. Atmospheric Environment, 41(2), pp. 360-368, 2007.

[3] Turpin, C. \& Harion, J.L., Numerical modeling of flow structures over various flat-topped stockpiles height: Implications on dust emissions. Atmospheric Environment, 43(35), pp. 5579-5587, 2009.

[4] Toraño, J., Rodriguez, R., Diego, I., Rivas, J. \& Pelegry, A., Influence of the pile shape on wind erosion cfd emission simulation. Applied Mathematical Modelling, 31(11), pp. 2487-2502, 2007.

[5] Diego, I., Pelegry, A., Torno, S., Toraño, J. \& Menendez, M., Simultaneous cfd evaluation of wind flow and dust emission in open storage piles. Applied Mathematical Modelling, 33(7), pp. 3197-3207, 2009.

[6] Toraño, J., Torno, S., Menendez, I.D.M. \& Gent, M., Dust emissions calculations in open storage piles protected by means of barriers, cfd and experimental tests. Environmental Fluid Mechanics, 9, pp. 493-507, 2009.

[7] Turpin, C. \& Harion, J.L., Effect of the topography of an industrial site on dust emissions from open storage yards. Environmental Fluid Mechanics, 2010.

[8] EDF R\&D, Code Saturne practical user's guide, 2011.

[9] Badr, T. \& Harion, J., Numerical modelling of flow over stockpiles: Implications on dust emissions. Atmospheric Environment, 39(30), pp. 5576$5584,2005$.

[10] Turpin, C., Amélioration des modèles de quantification des émissions particulaires diffuses liées l'érosion éolienne de tas de stockage de matières granulaires sur sites industriels. Ph.D. thesis, University of Valenciennes, 2010.

[11] Harion, J.L., Evrard, C., Furieri, B., Lecocq, V., Guinard, A., Ranty, C., Michel, O. \& Alac, G., Amélioration et validation de la méthode de quantification des émissions diffuses de particules de plein air sur sites, ADEME-ARMINES-LECES-ArcelorMittal, Convention ADEME-ARMINES 08-74-C0128., 2010. 